\title{
Correction to: The cross-sectional average length of healthy life (HCAL): a measure that summarizes the history of cohort health and mortality
}

Markus Sauerberg ${ }^{1,2^{*}}$, Michel Guillot ${ }^{3,4}$ and Marc Luy ${ }^{1,2}$

\section{Correction to: Popul Health Metrics 18, 21 (2020) https://doi.org/10.1186/s12963-020-00220-5}

Following publication of the original article [1], it was several errors were reported in the main text of the article, and also in Table 1.

The heading of Table 1 was missing the symbol ' $\pi$ ', and similarly it was missing in the body of the Table for the rows indicating the total prevalence of being unhealthy for the nine European countries. The corrected Table 1 is given below.

In the Results section the following sentences have been corrected, with the text highlighted in bold added.

- For example, Italian males spend $81.06 \%$ of their total life years in good health on the basis of HCAL, whereas the proportion of healthy life years is only $78.44 \%$ on the basis of $\mathrm{HE}$.
- As mentioned above, differences between HE and HCAL in relative terms result from the relative difference between the $p(x, t)$ function and the $p_{c}(x$, $t-x$ ) function, leading to an (un)favorable agespecific weighting scheme for women.

In the Discussion section the following sentence has been corrected, with the corrected text highlighted in bold originally erroneously reversed.

- The ratio of HE/LE respective $\mathrm{HCAL} / \mathrm{CAL}$ is particularly relevant in this context because it shows the relative share of healthy life years on total life years.

The original text also contained some minor typographical errors which have been amended. The original article has been updated.

* Correspondence: markus.sauerberg@oeaw.ac.at

${ }^{1}$ Vienna Institute of Demography, Austrian Academy of Sciences, Vienna, Austria

${ }^{2}$ Wittgenstein Centre for Demography and Global Human Capital (IIASA, OeAW, University of Vienna), Vienna, Austria

Full list of author information is available at the end of the article licence and your intended use is not permitted by statutory regulation or exceeds the permitted use, you will need to obtain permission directly from the copyright holder. To view a copy of this licence, visit http://creativecommons.org/licenses/by/4.0/ The Creative Commons Public Domain Dedication waiver (http://creativecommons.org/publicdomain/zero/1.0/) applies to the data made available in this article, unless otherwise stated in a credit line to the data. 
Table 1 Total sample size N (unweighted) and total prevalence of being unhealthy $\pi$ (weighted) for nine European countries from 2008 to 2014

\begin{tabular}{|c|c|c|c|c|c|c|c|c|c|}
\hline & & & \multicolumn{7}{|c|}{ EU-SILC Survey Year } \\
\hline & & & 2008 & 2009 & 2010 & 2011 & 2012 & 2013 & 2014 \\
\hline \multirow[t]{4}{*}{ Denmark } & Females & $N$ & 3019 & 3101 & 3072 & 2655 & 2737 & 2784 & 2959 \\
\hline & Males & & 2758 & 2765 & 2794 & 2477 & 2552 & 2635 & 2798 \\
\hline & Females & $\pi$ & 30.71 & 30.66 & 29.59 & 31.58 & 30.68 & 31.70 & 31.37 \\
\hline & Males & & 23.65 & 24.42 & 23.46 & 21.93 & 27.24 & 26.72 & 28.57 \\
\hline \multirow[t]{4}{*}{ Finland } & Females & $N$ & 5175 & 5050 & 5423 & 4512 & 4854 & 5383 & 5418 \\
\hline & Males & & 5128 & 4912 & 5267 & 4586 & 4885 & 5371 & 5405 \\
\hline & Females & $\pi$ & 34.99 & 36.17 & 37.11 & 37.56 & 40.82 & 47.84 & 39.28 \\
\hline & Males & & 29.18 & 29.51 & 29.18 & 30.82 & 32.67 & 40.61 & 30.97 \\
\hline \multirow[t]{4}{*}{ France } & Females & $N$ & 10,473 & 10,568 & 10,944 & 11,132 & 11,771 & 10,803 & 11,113 \\
\hline & Males & & 9535 & 9545 & 9944 & 10,164 & 10,742 & 9782 & 9985 \\
\hline & Females & $\pi$ & 25.08 & 26.61 & 27.23 & 27.10 & 26.86 & 26.99 & 27.01 \\
\hline & Males & & 21.07 & 21.36 & 23.05 & 22.47 & 22.84 & 22.67 & 22.41 \\
\hline \multirow[t]{4}{*}{ Italy } & Females & $N$ & 22,635 & 22,072 & NA & 20,392 & 20,325 & 19,039 & 20,409 \\
\hline & Males & & 20,741 & 20,087 & NA & 18,564 & 18,475 & 17,324 & 18,435 \\
\hline & Females & $\pi$ & 31.19 & 30.35 & NA & 31.59 & 32.49 & 32.95 & 31.75 \\
\hline & Males & & 23.62 & 22.97 & NA & 24.07 & 26.21 & 26.69 & 25.93 \\
\hline \multirow[t]{4}{*}{ Germany } & Females & $N$ & 12,579 & 12,323 & 12,191 & 12,497 & 12,181 & 11,671 & 11,715 \\
\hline & Males & & 11,547 & 11,363 & 11,211 & 11,548 & 11,272 & 10,709 & 10,780 \\
\hline & Females & $\pi$ & 36.44 & 35.56 & 35.50 & 36.14 & 38.03 & 38.80 & 40.17 \\
\hline & Males & & 33.68 & 32.82 & 32.42 & 33.52 & 34.87 & 34.83 & 36.78 \\
\hline \multirow[t]{4}{*}{ Netherlands } & Females & $N$ & 5667 & 5274 & 5494 & 5679 & 5479 & 5384 & 5464 \\
\hline & Males & & 4648 & 4443 & 4628 & 4794 & 4667 & 4706 & 4680 \\
\hline & Females & $\pi$ & 34.14 & 33.94 & 33.50 & 34.79 & 35.91 & 39.69 & 36.74 \\
\hline & Males & & 24.68 & 25.81 & 26.35 & 23.60 & 24.73 & 27.44 & 24.95 \\
\hline \multirow[t]{4}{*}{ Norway } & Females & $N$ & 2632 & 2587 & 2457 & 2054 & 2820 & 2838 & 3490 \\
\hline & Males & & 2853 & 2762 & 2704 & 2343 & 3158 & 3107 & 3782 \\
\hline & Females & $\pi$ & 21.79 & 21.39 & 20.03 & 26.45 & 17.52 & 22.07 & 21.69 \\
\hline & Males & & 13.40 & 14.87 & 14.87 & 18.54 & 11.99 & 14.16 & 12.49 \\
\hline \multirow[t]{4}{*}{ Sweden } & Females & $N$ & 3834 & 3891 & 3713 & 3512 & 3482 & 3165 & 2933 \\
\hline & Males & & 3612 & 3649 & 3451 & 3193 & 3136 & 3025 & 2834 \\
\hline & Females & $\pi$ & 29.29 & 26.91 & 26.66 & 27.88 & 27.28 & 27.22 & 16.95 \\
\hline & Males & & 21.44 & 19.45 & 20.01 & 21.27 & 20.82 & 20.10 & 10.55 \\
\hline \multirow[t]{4}{*}{ UK } & Females & $N$ & 8725 & 8081 & 7827 & 7728 & 9688 & 9716 & 9466 \\
\hline & Males & & 7816 & 7278 & 6970 & 6949 & 8648 & 8692 & 8437 \\
\hline & Females & $\pi$ & 20.74 & 21.68 & 22.23 & 23.55 & 23.63 & 23.16 & 24.52 \\
\hline & Males & & 18.22 & 18.91 & 19.18 & 19.42 & 19.70 & 19.85 & 21.43 \\
\hline
\end{tabular}

Source: EU-SILC data (own calculations)

Author details

${ }^{1}$ Vienna Institute of Demography, Austrian Academy of Sciences, Vienna, Austria. ${ }^{2}$ Wittgenstein Centre for Demography and Global Human Capital (IIASA, OeAW, University of Vienna), Vienna, Austria. ${ }^{3}$ Population Studies Center, University of Pennsylvania, Philadelphia, PA, USA. ${ }^{4}$ French Institute for Demographic Studies (INED), Paris, France.
Published online: 21 April 2021

\section{Reference}

1. Sauerberg M, Guillot M, Luy M. The cross-sectional average length of healthy life (HCAL): a measure that summarizes the history of cohort health and mortality. Popul Health Metrics. 2020;18(1):21. https://doi.org/10.1186/ s12963-020-00220-5. 